\title{
Ternary alkali metal zinc antimonides and bismuthides: hydride synthesis and in- situ X-ray diffraction study
}

\author{
J Zaikina ${ }^{1}$, T Cox ${ }^{1}$, V Gvozdetskyi ${ }^{1}$ \\ ${ }^{1}$ Department of Chemistry lowa State University, Ames, IA \\ yzaikina@iastate.edu
}

Materials discovery can be accelerated by the development of synthesis methods and in-situ characterization techniques allowing for the rapid "screening" of multicomponent systems. However, the sluggish kinetics of solidstate reactions entails the necessity of high temperatures and long annealing times, often leading to the stabilization of the thermodynamically stable products. Thus, new synthetic methods using unconventional reactive precursors must be developed to overcome limitations of traditional solid-state synthesis. Such methods will allow exploration of systems where starting materials have drastically different reactivity. We are interested in unconventional synthesis methods toward solid-state materials, using reactive, salt-like precursors. The chosen synthetic method utilizes alkali metal-hydride precursors and remedies the problem of insufficient mixing of the starting materials with ductile alkali metals, leading to faster kinetics and reduced annealing times. In addition, this route allows for targeted synthesis of specific compositions, and thus, fine tuning of physical properties. Such a synthetic approach could help bridge the gap between theoretical prediction and experimental synthesis of new materials, allowing for rapid exploration of potentially rich phase space in the ternary systems containing an alkali metal. Utilizing this synthesis method, new ternary alkali-metal zinc antimonides and bismuthides in the Na-Zn-Sb, Na-Zn-Bi, and K$\mathrm{Zn}-\mathrm{Sb}$ systems have been synthesized and characterized. These new compounds are hardly accessible via traditional solid-state route, because of the ductility and high reactivity of the alkali metal and side reactions resulting in inhomogeneous samples. The in-situ powder X-ray diffraction allowed to assess the thermal stability of the compounds and to further optimize synthesis of the phase pure samples. Additionally, in-situ powder X-ray diffraction helped to identify and subsequently synthesize high-temperature phases, which are hidden otherwise.

Acta Cryst. (2020). A76, a204 